\title{
ON CLOSED SETS OF ORDINALS
}

\author{
HARVEY FRIEDMAN ${ }^{1}$
}

\begin{abstract}
We prove that every stationary set of countable ordinals contains arbitrarily long countable closed subsets.
\end{abstract}

Call a set $A$ of ordinals closed if and only if every nonempty subset of $A$ which has an upper bound in $A$ has its least upper bound in $A$. It is well known that there are $B \subset \omega_{1}$ such that neither $B$ nor $\omega_{1}-B$ contains an uncountable closed subset. A consequence of what we prove here is that for every $B \subset \omega_{1}$, either $B$ or $\omega_{1}-B$ contains arbitrarily long countable closed subsets.

Call a set $A$ of ordinals $\kappa$-stationary if and only if $A \subset \kappa$ and $A$ intersects every closed subset of $\kappa$ of power $\kappa$. We can restate the above wellknown theorem as follows: There is an $A$ such that $A$ and $\omega_{1}-A$ are both $\omega_{1}$-stationary. ${ }^{2}$

We will prove here that every $\omega_{1}$-stationary set contains arbitrarily long, countable, closed subsets.

Is there a cardinal $\kappa$ such that for all $A \subset \kappa$, either $A$ or $\kappa-A$ contains an uncountable closed subset? Is this true for $\kappa=\omega_{2}$ ? Karel Prikry and the author noticed that, in any case, the statement for $\kappa=\omega_{2}$ cannot be proved true in $\mathrm{ZFC}^{3}$

THEOREM. Every $\omega_{1}$-stationary set contains arbitrarily long countable closed subsets.

Proof. Let $A$ be $\omega_{1}$-stationary. We prove by induction on $\alpha<\omega_{1}$ that $A$ has a closed subset of length $\alpha$. Let the induction hypothesis be that

Received by the editors November 30, 1972.

AMS (MOS) subject classifications (1970). Primary 04A20; Secondary 02K02, 02K35.

Key words and phrases. Ordinals, cardinals.

${ }^{1}$ The writing of this paper was partially supported by NSF grant GP-34091X. The Theorem was obtained in 1968.

${ }^{2}$ In fact, Solovay [1] proves that for uncountable regular cardinals $\kappa$, every $\kappa$ stationary set is the union of $\kappa$ disjoint $\kappa$-stationary sets.

${ }^{3}$ By adding an $f: \omega_{2} \rightarrow\{0,1\}$ generic with respect to the partial ordering of countable partial $g: \omega_{2} \rightarrow\{0,1\}$. If the ground model satisfies $Z F C+2^{\omega}=\omega_{1}$, then in the forcing extension cardinals are preserved, $\{\alpha: f(\alpha)=1\}$ and $\{\alpha: f(\alpha)=0\}$ contain no uncountable closed subsets, and $2^{\omega}=\omega_{1}$ holds.

(c) American Mathematical Society 1974 
for all $\beta<\alpha$ and for each $\gamma<\omega_{1}$, there is a closed subset $B \subset A$ of length $\beta$, all of whose elements are $>\gamma$.

Case 1. $\alpha$ is a limit ordinal $<\omega_{1}$. Choose $\beta_{0}<\beta_{1}<\cdots<\alpha$, with $\sup _{n}\left(\beta_{n}\right)=\alpha$. Let $\gamma<\omega_{1}$. By the induction hypothesis, let $B_{0} \subset A, B_{0}$ of length $\beta_{0}+1, B_{0}$ closed, $\left(\forall \beta \in B_{0}\right)(\beta>\gamma)$. Let $B_{n+1} \subset A, B_{n+1}$ of length $\beta_{n+1}+1, B_{n+1}$ closed, $\left(\forall \beta \in B_{n+1}\right)\left(\beta>\sup \left(B_{n}\right)\right)$. Then set $B=\bigcup_{n} B_{n}$. $B$ has the desired properties.

Case 2. $\alpha=\delta+2, \alpha<\omega_{1}$. Let $\gamma<\omega_{1}$. By the induction hypothesis, let $B_{0} \subset A$ be closed, of length $\delta+1$, and $\left(\forall \beta \in B_{0}\right)(\beta>\gamma)$. Let $\lambda \in A$ with $\lambda>\sup \left(B_{0}\right)$. Put $B=B_{0} \cup\{\lambda\}$. $B$ has the desired properties.

Case 3. $\alpha=\lambda+1, \lambda$ a limit ordinal $<\omega_{1}$. Let $\lambda_{0}<\lambda_{1}<\cdots<\lambda$, $\sup _{n}\left(\lambda_{n}\right)=\lambda$. Let $\gamma<\omega_{1}$. By the induction hypothesis, define a sequence of sets $B_{\xi}, \xi<\omega_{1}$, such that

(a) $\left(\forall \beta \in B_{0}\right)(\beta>\gamma)$

(b) if $\xi_{1}<\xi_{2}$ then $\left(\forall \beta_{1} \in B_{\xi_{1}}\right)\left(\forall \beta_{2} \in B_{\xi_{2}}\right)\left(\beta_{1}<\beta_{2}\right)$

(c) each $B_{\xi}$ is a closed subset of $A$ of length $\lambda$.

Define $f: \omega_{1} \rightarrow \omega_{1}$ by $f(\xi)=\sup \left(\bigcup_{\sigma<\xi} B_{\sigma}\right)$. Note that the range of $f$ on limit ordinals is an uncountable closed set. Since $A$ is stationary, let $\tau$ be a countable limit ordinal with $f(\tau) \in A$. Choose $\tau_{0}<\tau_{1}<\cdots<\tau$ with $\sup _{n}\left(\tau_{n}\right)=\tau$. Let $C_{n}$ be the first $\lambda_{n}+1$ elements of $B_{\tau_{n}}$. Then set $B^{*}=\bigcup_{n} C_{n}$. $B^{*}$ is a closed subset of $A$ of length $\lambda$, and $\sup \left(B^{*}\right)=f(\tau) \in A$. Hence $B=B^{*} \cup\{f(\tau)\}$ is a closed subset of length at least $\alpha$, all of whose elements are $>\gamma$, and we are done.

The referee has kindly forwarded the following remarks concerning the problems raised on the first page of this paper.

Let us say that a cardinal $K>\omega$ has the property $F$ (briefly, $F(K)$ ) if for every subset $A$ of $K$ either $A$ or $K-A$ contains a closed subset of order type $\omega_{1}$.

(1) Silver has shown that the Jensen principle $\square_{\omega_{1}}$ implies $\neg F\left(\omega_{2}\right)$. Since $\neg \square \square_{\omega_{1}} \rightarrow$ " $\omega_{2}$ is Mahlo in $L$ " this gives a lower bound on the prooftheoretic strength of $\mathrm{ZFC}+F\left(\omega_{2}\right)$.

(2) Silver has also observed that in any Cohen extension of any model $M$ of ZFC obtained by generically collapsing $\omega_{1}^{M}$ to $\omega, F(K)$ fails for all uncountable $M$-cardinals $K$. (For $A$ take $\left\{\alpha: c f^{M}(\alpha)=\omega\right.$ and $\left.\alpha<K\right\}$.)

(3) Solovay has generalized Silver's proof in (1) above to show that, in $L, F(K)$ fails for all cardinals $K>\omega$.

Karel Prikry has informed the author that he has independently shown that $F(K)$ fails for all cardinals $K>\omega$, in $L$. 


\section{REFERENCE}

1. R. M. Solovay, Real-valued measurable cardinals, Axiomatic Set Theory (Proc. Sympos. Pure Math., vol. 13, Part I, Univ. California, Los Angeles, Calif., 1967), Amer. Math. Soc., Providence, R.I., 1971, pp. 397-428. MR 45 \#55.

Department of Mathematics, Stanford University, Stanford, California 94305

Department of Mathematics, State University of New York at Buffalo, AMHERST, NeW YORK 14226 